\title{
Comparison of Remote Pressure and Vibrotactile Feedback for Prosthetic Hand Control
}

\author{
Camilo Tejeiro, Cara E. Stepp, Member IEEE, Mark Malhotra, Eric Rombokas, Member, IEEE, and Yoky Matsuoka, \\ Member, IEEE
}

\begin{abstract}
Prosthetic hand users may benefit from incorporation of sensory feedback, but the optimal methods of providing such feedback are still unknown. The effect on object manipulation of adding vibrotactile and pressure feedback at remote body sites to visual feedback was assessed in 8 participants using a virtual and robotic interface. Both pressure and vibrotactile feedback improved some aspects of task performance when compared to visual feedback alone; however, no difference in object manipulation performance was seen between pressure and vibrotactile feedback.
\end{abstract}

Upper limb prosthetics; sensory substitution; pressure feedback; vibrotactile feedback

\section{INTRODUCTION}

The field of hand prosthetics has expanded rapidly with improvements in hand dexterity as well as development of new mechanisms for interfacing with the body for control. These developments have allowed for the introduction of commercially available EMG-actuated prostheses with increased degrees of freedom and more natural control (e.g. Otto Bock's SensorHand Speed and Touch Bionics' i-LIMB Hand). These advances in prosthetics have been tailored towards fulfilling the needs expressed by amputees to improve their quality of life [1, 2]. However, amputees have also expressed dissatisfaction with the heavy dependence on visual feedback required to operate current prosthetics $[1,2]$. Additional sensory feedback could improve manipulation in those cases where visual feedback is not sufficient (e.g. in dark environments or handling fragile objects) [3].

There are currently several prototypes of prosthetic hands with force sensors implemented into their fingertips [4, 5]. However, the question that still remains is how to optimally provide force feedback to users.

Manuscript received January 14, 2012.

C. Tejeiro is with the Department of Computer Science \& Engineering, University of Washington, Seattle, WA 98195 USA (e-mail:ctejeiro@ee.washington.edu).

C. E. Stepp is with the Departments of Computer Science \& Engineering and Rehabilitation Medicine, University of Washington, Seattle, WA 98195 USA (E-mail:cstepp@alum.mit.edu).

M. Malhotra is with the Department of Computer Science \& Engineering, University of Washington, Seattle, WA 98195 USA

(phone: 650-224-9264; fax: 206-543-2969 ;

E-mail:malhotra@standfordalumni.org).

E. Rombokas is with the Department of Electrical Engineering, University of Washington, Seattle, WA 98195 USA

(E-mail:rombokas@cs.washington.edu).

Y. Matsuoka is with the Department of Computer Science \& Engineering, University of Washington, Seattle, WA 98195 USA (e-mail:yoky@cs.washington.edu).
To answer this question several considerations have to be taken into account, including: stimulation discomfort, the ability of users to relate feedback to force, invasiveness, technical constraints, and pragmatic factors such as cost and weight. Several modalities have been suggested such as: electrocutaneous, acoustic, direct nerve, pressure and vibrotactile stimulation [6-11]. Both pressure and vibrotactile stimulation are non-invasive, and thus have the potential for implementation in a wide array of users.

Vibrotactile stimulation at a remote body site has been shown to be a viable feedback mechanism when implemented for hand prostheses [4, 12, 13]. Substituting remote pressure feedback for forced sensed on prosthetic fingertips has not been as widely researched, despite the natural correspondence between force and pressure modalities. In 1993, Patterson \& Katz used a betweensubjects design to compare pressure and vibrotactile feedback during a force matching task that involved gripping with a robotic arm. The study found a trend for larger relative error in the 5 participants using vision plus vibration relative to the error of the 5 participants using pressure plus vision [14]. However, only 5 participants were recorded in each of these conditions and statistical testing was not attempted.

The purpose of this study was to utilize a within-subjects design (repeated measures) to characterize the effects of feedback type (vision alone, vibrotactile plus vision, and pressure plus vision) on the performance of a virtual object manipulation task. We hypothesized that participants would be able to achieve increased performance with the addition of pressure and vibrotactile feedback relative to visual feedback alone. Experiments indicate that participants have an increased ability to control applied force with both vibrotactile and pressure-based sensory substitution. These results could pave the road toward the implementation of sensory feedback systems in current commercial prosthetics.

\section{METHODS}

For this experiment, participants interacted with a virtual environment using their index finger. They were asked to apply a normal force on a box on the left of the virtual environment in order to drag the box to a target in the right hand side of the environment as quickly as possible without breaking it. This task was developed based on the great difficulty experienced by myoelectric hand prosthetic users while delivering forces to fragile objects [3] and was specifically designed to be difficult enough to unmask 
potential differences in motor performance as a result of different feedback modalities.

\section{A. Virtual Elements and Visual Feedback}

The virtual environment was programmed in $\mathrm{C}++$, with graphics rendered by OpenGL. The environment consisted of a draggable box, a target area, and a marker indicating the position of the participant's finger in the virtual environment. There were two types of draggable boxes, identified by color, blue or red, each with different stiffnesses. During experimentation, the box began on the left side of the virtual environment. Participants were asked to apply a virtual force to the top of the box to overcome friction and drag the box towards the target.
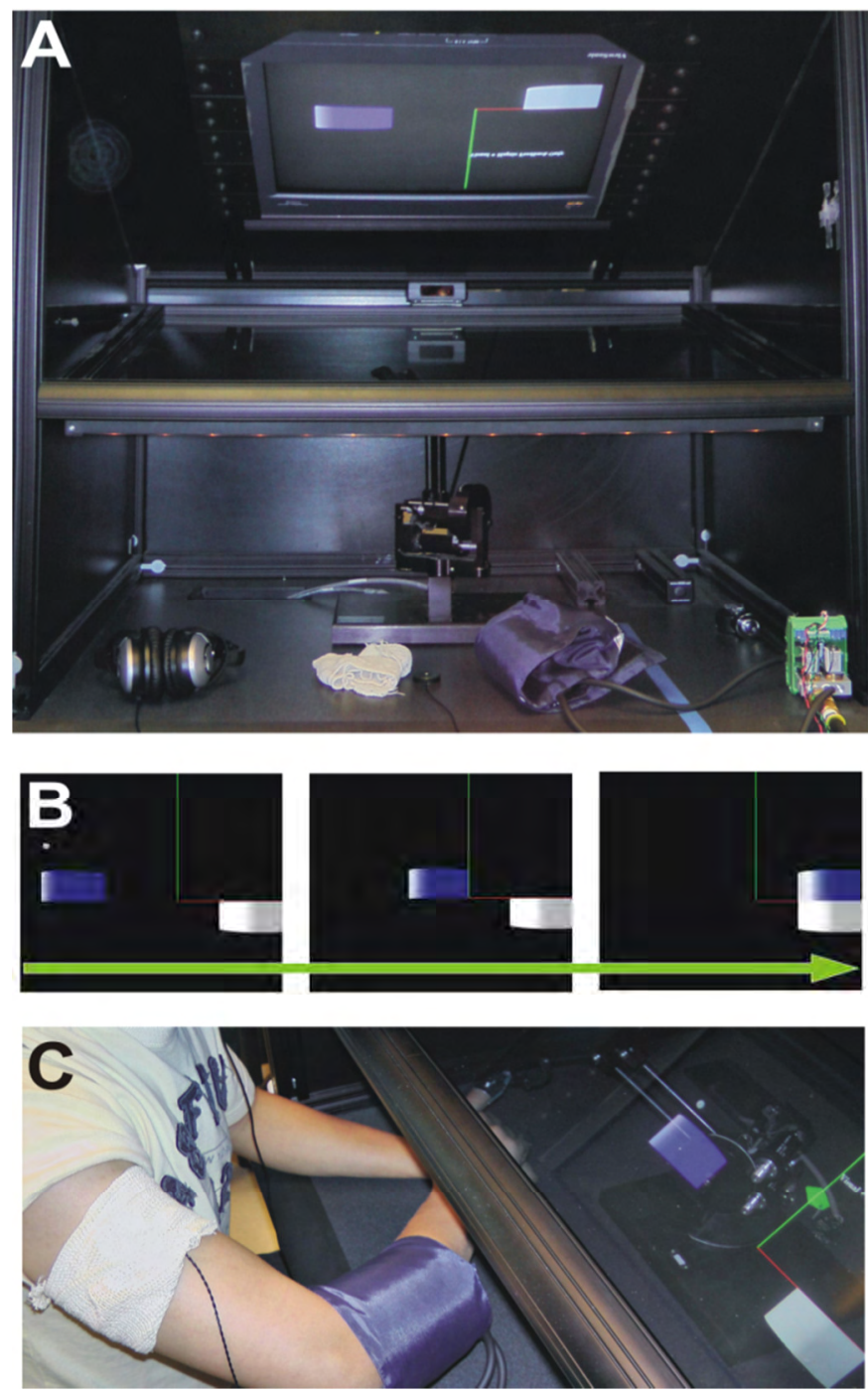

Fig 1. Experiment Set Up. Panel A: Elements used for the experiment, from left to right: headphones, tactor and bandage, Phantom, pressure cuff, and pressure regulator. Panel B: Screenshots of the motor task. The goal was to drag the movable box to the target as quickly as possible without breaking it. The marker was hidden after it made contact with the box. Panel C: The tactor and pressure cuff were located on the right arm of each participant, and the participants interacted with the environment using their right index finger.

\section{B. Participant Interaction with the Virtual Environment}

The movement of the participant's finger was sensed using a PHANTOM Premium 1.0 robotic device (Sensable Technologies, Inc, Woburn, MA). Participants placed their right index finger into a small cuff that was attached to the PHANTOM end effector. The PHANTOM was exclusively used to trace the three dimensional movements and did not provide any kind of force feedback.

The stiffness of the draggable boxes was defined in terms of the vertical displacement of the virtual finger by the piecewise functions in equations 1 and 2.

$F_{\text {blue }}=\left\{\begin{array}{cc}(0.34 \mathrm{~N} / \mathrm{cm}) x, & \text { if } x<1.7 \mathrm{~cm} \\ \left(4.65 \mathrm{~N} / \mathrm{cm}^{2}\right) x^{2}-(14.33 \mathrm{~N} / \mathrm{cm}) x+11.55, & \text { if } x>1.7 \mathrm{~cm}\end{array}\right.$

$$
F_{\text {red }}=\left\{\begin{array}{cc}
(0.68 \mathrm{~N} / \mathrm{cm}) x, & \text { if } x<1.7 \mathrm{~cm} \\
\left(9.59 \mathrm{~N} / \mathrm{cm}^{2}\right) x^{2}-(30.67 \mathrm{~N} / \mathrm{cm}) x+25.56, & \text { if } x>1.7 \mathrm{~cm}
\end{array}\right.
$$

These functions were based on force-displacement curves fit to empirical data from pushing on a disposable plastic cup. The forces described $\left(F_{\text {blue }}, F_{\text {red }}\right)$ were the virtual normal forces on the blue and the red box, respectively, and $x$ represents the virtual vertical displacement in centimeters. Both the linear and the quadratic characteristics of the functions were decreased for the blue box relative to the red box.

The vertical force required to move the boxes and overcome friction $\left(F_{\text {move }}\right)$ was set at 1.2 times the force at $x$ $=1.7 \mathrm{~cm}$. For both boxes, the force to break the box $\left(F_{\text {break }}\right)$ was defined as $0.75 \mathrm{~N}$ greater than $F_{\text {move }}$. Therefore, to move the box the participant had to apply a force in between $F_{\text {move }}$ and $F_{\text {break }}$. Since the stiffnesses of the blue box and the red box were different, the $0.75 \mathrm{~N}$ window for moving the boxes created different allowable vertical displacements for the boxes (1.6 $\mathrm{mm}$ for the red box and $2.7 \mathrm{~mm}$ for the blue box).

Momentum was not considered in the system dynamics. The marker was occluded during penetration of the box, and deformations of the box were not shown.

\section{Experimental Design}

Participants were 8 healthy adults ( 8 right handed; 5 male; 3 female; mean age $=20.1$ years; $\mathrm{SD}=3.0$ years). Informed consent in compliance with the Institutional Review Board of the University of Washington was obtained from all participants. Each participant performed 144 trials in a single session over $1.5-2$ hours including breaks. Trials were presented in 6 blocks of 24 trials randomized as a function of box type (red or blue), cognitive task (off or on), and feedback type (vision alone, vision plus vibrotactile, and vision plus pressure). Each block of 24 trials included 2 repetitions of each feedback condition. Participants sat in front of the virtual environment with their right arms extended to move freely about the 3D workspace. Trials ended if the box was broken or if it reached the target. To prevent fatigue participants were required to take a 5 minute 
break halfway through experimentation; they were also encouraged to take as many breaks as needed between trials.

During some trials a cognitive task was performed by the participants simultaneously with the motor task. The cognitive task consisted of listening to a string of 16 random numbers and indicating verbally when a set of repeated numbers with one number in between was presented [15]. All the participants practiced this task before starting the experiment. Noise cancelling earphones (Bose, Framingham, MA) were used to present the stimuli for this task.

\section{Vibrotactile Feedback}

During trials using vibrotactile feedback, stimulation was delivered using a C2 tactor (Engineering Acoustics, Inc). We chose to incorporate the $\mathrm{C} 2$ tactor because its linear actuation allows for complex stimulation paradigms and dissociated changes in frequency and amplitude. The tactor was mounted on the lateral side of the right upper arm of the subject, and elastic kept the tactor in place. Feedback consisted of a $250 \mathrm{~Hz}$ sine wave with mechanical amplitude ranging from $0-400 \mu \mathrm{m}$, proportional to the applied virtual force $\left(F_{\text {red }}\right.$ or $\left.F_{\text {blue }}\right)$. Human skin has been shown to be most sensitive to vibration at this frequency $[16,17]$. However this frequency is also within the range of audition; therefore a low level broad-band noise was used to mask the vibration. This noise was delivered using the same headphones that were used to deliver the stimuli for the cognitive task.

\section{E. Pressure Feedback}

A pneumatic system was developed to control the pressure in a cuff placed on the upper arm of the subjects, similar to a sphygmomanometer. The pneumatic system was implemented using an air compressor (Central Pneumatic, three gallon oil-less air compressor, Model 95275) as the air supply, which was calibrated to deliver a constant $91.7 \mathrm{kPa}$ to the pressure regulator (Marsh Bellofram Electro-Pneumatics, series T-3110). The pressure regulator maintained a pressure in the arm cuff that was linearly proportional to the virtual force, ranging between $2.66 \mathrm{kPa}(20 \mathrm{mmHg})-11.97 \mathrm{kPa}(90 \mathrm{mmHg})$. The pressure range implemented was strong enough to be easily perceived, but not strong enough to conflict with the participant's blood circulation or cause discomfort. The pressure regulator in this set up was chosen due to its ability to operate on an analog input and deliver low pressure ranges covering the human systolic pressure $(90-120$ $\mathrm{mmHg}$ ).

\section{F. Data Analysis}

The performance of the participants was evaluated using the total box displacement towards the target and the average box velocity (the box displacement normalized by the trial time). MATLAB (Mathworks, Natick MA) was used to calculate box displacement and velocity for each trial. Statistical analysis was performed using Minitab Statistical Software (Minitab Inc., State College, PA). A 4 factor repeated measures analysis of variance (ANOVA) was performed to assess the effects of feedback (vision alone, vision plus vibrotactile, vision plus pressure), cognitive task (on, off), presentation order (block), and box (red, blue), as well as the interactions of feedback $\times$ block, cognitive task $\times$ feedback, and block $\times$ cognitive task on box displacement and velocity. Post hoc two-sided Tukey's Simultaneous tests were performed as appropriate. Statistical analyses were performed using an alpha level of 0.05 for significance.

\section{RESULTS}

Out of 1152 combined trials, participants were able to successfully move the box to the target 85 times $(7.4 \%$ of attempts). The average displacement achieved during these successful attempts was the full range of the task $(30 \mathrm{~cm})$ and the average box velocity was $0.58 \mathrm{~cm} / \mathrm{s}(\mathrm{SE}=0.03$ $\mathrm{cm} / \mathrm{s})$. During unsuccessful attempts, the average displacement achieved was $3.45 \mathrm{~cm}(\mathrm{SE}=0.20 \mathrm{~cm})$ and the average box velocity was $0.16 \mathrm{~cm} / \mathrm{s}(\mathrm{SE}=0.01 \mathrm{~cm} / \mathrm{s})$.

An ANOVA showed significant effects on box displacement of feedback, box, and block (see Table I). A simultaneous cognitive task did not show a significant effect on the displacement, and no significant interactions were found between the factors. Post hoc testing indicated that both vision plus vibrotactile and vision plus pressure feedback resulted in increased box displacement relative to vision alone (see fig. 2). However, no significant difference was seen between the displacement during vision plus vibrotactile and vision plus pressure feedback. Post hoc testing as a function of block indicated a training effect, with increases in box displacement as a function of block. Figure 2 shows the significant comparisons. As expected due to the variable displacement allowance, the trials using the red box resulted in significantly decreased displacement relative to the blue box.

An ANOVA showed significant effects on average box velocity of box and block (see Table II). The feedback modality and simultaneous cognitive task did not show a significant effect $(p<0.05)$ on the average box velocity, and no significant interactions were found between the factors. However, a trend was seen for an effect of feedback, with $p=0.051$. Post hoc testing as a function of block indicated a training effect, with increases in average box velocity as a function of block. Figure 2 shows the significant comparisons. Trials using the red box resulted in significantly decreased velocity relative to the blue box.

TABLE I

ANOVA RESULTS FOR BOX DISPLACEMENT

\begin{tabular}{lrrr}
\hline \multicolumn{1}{c}{ Effect } & DF & \multicolumn{1}{c}{ F } & \multicolumn{1}{c}{$p$} \\
\hline Feedback & 2 & 11.8 & $<0.001$ \\
Cognitive Task & 1 & 1.2 & 0.271 \\
Presentation Order & 5 & 18.2 & $<0.001$ \\
Box & 1 & 179.0 & $<0.001$ \\
Feedback $\times$ Cognitive Task & 2 & 0.5 & 0.623 \\
Feedback $\times$ Presentation Order & 10 & 1.2 & 0.265 \\
Cognitive Task $\times$ Presentation Order & 5 & 1.0 & 0.390 \\
\hline \hline
\end{tabular}


TABLE II

ANOVA RESULTS FOR BOX VELOCITY

\begin{tabular}{lrrr}
\hline \multicolumn{1}{c}{ Effect } & DF & \multicolumn{1}{c}{ F } & \multicolumn{1}{c}{$p$} \\
\hline Feedback & 2 & 3.0 & 0.051 \\
Cognitive Task & 1 & 0.8 & 0.363 \\
Presentation Order & 5 & 30.5 & $<0.001$ \\
Box & 1 & 276.5 & $<0.001$ \\
Feedback $\times$ Cognitive Task & 2 & 0.75 & 0.472 \\
Feedback $\times$ Presentation Order & 10 & 0.94 & 0.500 \\
Cognitive Task $\times$ Presentation Order & 5 & 1.79 & 0.112 \\
\hline \hline
\end{tabular}
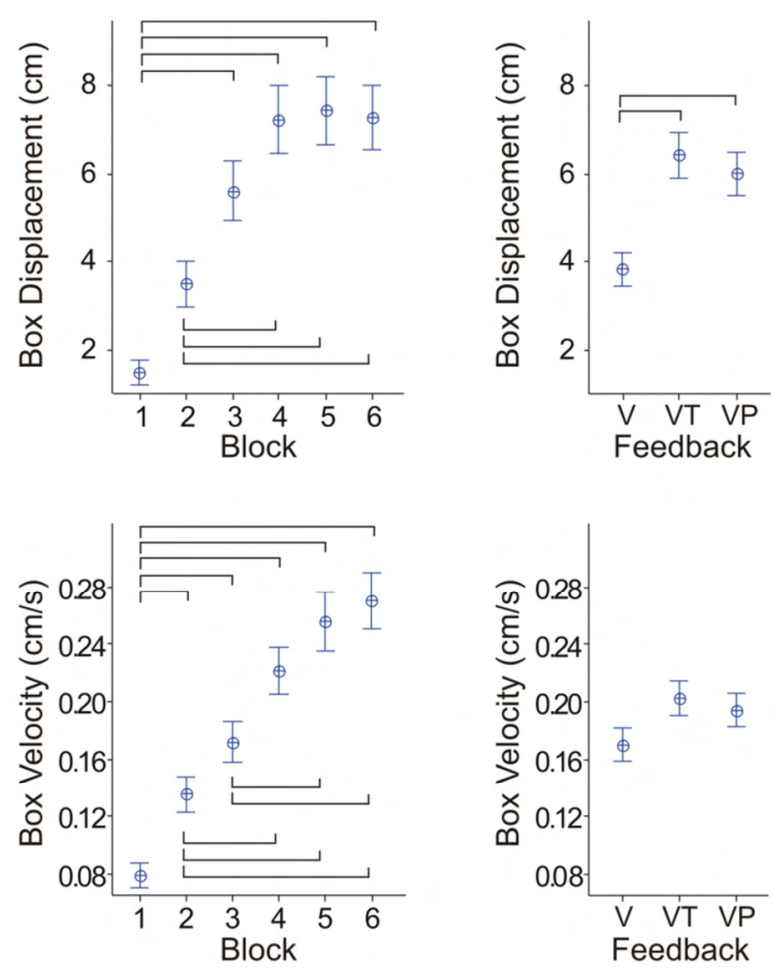

Fig 2. Experimental Results. Upper panels show box displacement as a function of block (left) and feedback modality (right; vision alone $=\mathrm{V}$, vision plus vibrotactile $=\mathrm{VT}$, vision plus pressure $=\mathrm{VP})$. Lower panels show average box velocity. Markers indicate the mean and error bars indicate $+/-1$ SE. Brackets show significant differences at the 5\% level.

\section{DISCUSSION}

\section{A. Effects of the Feedback Modality}

Our results show a significant effect of the use of both vibrotactile and pressure feedback on box displacement. This demonstrates the utility of augmentative force feedback for the task, over visual feedback alone.

Patterson \& Katz found an average relative error while using vision plus vibration of $8 \%$ in five participants relative to an average error using vision plus pressure of $6 \%$ in another five participants [14]. In their study, vision alone also resulted in a relative error of $8 \%$. They were not able to make a statistical recommendation since only 5 participants were recorded in each of these conditions. In our participants, the addition of either vibration or pressure to visual feedback did significantly increase the average displacement of the box. Contrary to their finding of a trend for increased task error during use of vision plus vibration relative to vision plus pressure, the results of our experiment show performance using vision plus vibration to match that obtained for vision plus pressure. This is consistent with a previous study in which haptic (pressure) was compared with vision and audition as sensory substitution modalities for force-related feedback during a virtual object manipulation task [18]. In that study, better initial performance was obtained using haptic feedback, but performance using vision or audition grew to equivalence with practice. This is promising for the use of vibrotactile feedback, which presents fewer technical hurdles to implement than pressure feedback, such as the need for a pressurized air tank. An alternative explanation for our study finding no difference in performance using pressure versus vibrotactile feedback is that neither of these two modalities was tailored to individual perceptual sensitivities. All participants were subject to the same feedback calibrations. A lack of calibration to individual participant sensitivies might have washed out the effects of feedback type.

The average box velocity did not show a significant effect of feedback modality. This is consistent with previous work comparing visual feedback alone to vision plus vibrotactile feedback, in which increases in displacement but not velocity were found during the use of the vibrotactile feedback [19].

This discrepancy between displacement and velocity results may be due to differences in the effect of feedback on task completion behavior. With augmentative feedback, the users are able to carefully slide the box, modulating the normal force along the way. With visual only feedback, however, a common strategy is to increase normal force until box displacement is observed, and to move quickly across the workspace while attempting to keep normal force constant. Additionally, trials in which the box is broken before any displacement occurs do not contribute to the average box velocity measure. A slow and careful trial resulting in a large displacement is more likely under the visual plus feedback conditions, contributing to a greater effect on displacement than velocity for those conditions. Future improvements to our experimental design will include delivering both vibrotactile and pressure feedback to differentiate upon contact and continuous manipulation of objects. The results from these studies will assess whether a multi-feedback approach substantially increases task performance as compared to using vibrotactile or pressure feedback alone.

\section{B. Effects of the Cognitive Task}

The presence of the cognitive task did not show a significant effect on box displacement or velocity. Our results show that the improvement due to augmentative feedback is preserved even in the face of a distracting cognitive task. This finding is promising for practical application of augmentative feedback for prosthetic hand users, who may need to perform challenging motor tasks in 
the face of other cognitive demands (e.g., a simultaneous conversation).

\section{Training Effects}

Our results indicate that task performance (both box displacement and velocity) drastically increased as a function of training time. However, no significant interaction was seen between these training effects and the feedback modality, suggesting that individuals were able to improve with practice regardless of which feedback modality was being used. Our previous work has shown that drastic improvements in performance using vibrotactile feedback are possible when individuals are able to train over multiple days [20]. Our future work will further explore vibrotactile and pressure feedback training effects over multiple days.

\section{Summary}

Both vibrotactile and pressure feedback resulted in significantly increased motor performance than using vision alone to complete the task. This study was undertaken to answer questions about the role of feedback modality in integrating augmentative force feedback to perform a difficult ( $7.4 \%$ success rate) motor task with the goal of improving prosthetic hand control. However, the participants in this study were intact individuals with kinesthetic sensation and the task was arguably more difficult than many tasks of daily living performed with prosthetic hands. The task was purposefully implemented to be difficult for participants to perform so that performance differences as a function of feedback modality would not be masked. In addition, we argue that more difficult and precise tasks such as the one implemented are most in need of augmentative feedback. Use of a virtual environment to implement the motor task allowed removal of cutaneous cues in healthy individuals, but kinesthetic cues about finger position were still available to participants. For the chosen task, fingertip force was the most relevant cue; however, we cannot remove the possibility that our results seen here in intact individuals do not extend to amputees. In our future work we will extend this paradigm to amputee participants and will incorporate tests of activities of daily living.

\section{REFERENCES}

[1] Pylatiuk, et al., "Results of an internet survey of myoelectric prosthetic hand users," Prosthet Orthot Int, vol. 31, pp. 362-70, Dec 2007.

[2] E. Biddiss, et al., "Consumer design priorities for upper limb prosthetics," disabil rehabil Assist Technol, vol. 2, pp. 346-57, Nov 2007.

[3] F. C. Huang, et al., "Visual and haptic feedback contribute to tuning and online control during object manipulation," J mot behav, vol. 39, pp. 179-93, May 2007.

[4] C. Pylatiuk, et al., "Progress in the development of a multifunctional hand prosthesis," Conf Proc IEEE Eng Med Biol Soc, vol. 6, pp. 4260-3, 2004.

[5] A. Zecca, et al., "Experimental analysis of the proprioceptive and exteroceptive sensors of an underactuated prosthetic hand," Advances in Rehabilitation Robotics, vol. 306, pp. 233-242, 2004.
[6] K. A. Kaczmarek, et al., "Electrotactile and vibrotactile displays for sensory substitution systems," IEEE Trans Biomed Eng, vol. 38, pp. 1-16, Jan 1991.

[7] G. F. Shannon, "A comparison of alternative means of providing sensory feedback on upper limb prostheses," Med Biol Eng, vol. 14, pp. 289-94, May 1976.

[8] R. R. Riso, "Strategies for providing upper extremity amputees with tactile and hand position feedback--moving closer to the bionic arm," Technol Health Care, vol. 7, pp. 401-9, 1999.

[9] R. N. Scott, et al., "Sensory-feedback system compatible with myoelectric control," Med Biol Eng Comput, vol. 18, pp. 65-9, Jan 1980.

[10] G. F. Shannon, "A myoelectrically-controlled prosthesis with sensory feedback," Med Biol Eng Comput, vol. 17, pp. 73-80, Jan 1979.

[11] P. J. Agnew and G. F. Shannon, "Training program for a myoelectrically controlled prosthesis with sensory feedback system," Am J Occup Ther, vol. 35, pp. 722-7, Nov 1981.

[12] M. C. Carrozza, et al., "Design of a Cybernetic Hand for Perception and Action," Biol Cybern, vol. 95, pp. 629-44, Dec 2006.

[13] L. Zollo, et al., "Biomechatronic design and control of an anthropomorphic artificial hand for prosthetic and robotic applications," IEEE-Asme Transactions on Mechatronics, vol. 12, pp. 418-429, Aug 2007.

[14] P. E. Patterson and J. A. Katz, "Design and evaluation of a sensory feedback system that provides grasping pressure in a myoelectric hand," J Rehabil Res Dev, vol. 29, pp. 1-8, Winter 1992.

[15] W. K. Kirchner, "Age differences in short-term retention of rapidly changing information," J Exp Psychol, vol. 55, pp. 352-8, Apr 1958.

[16] R. T. Verrillo, "Vibration sensation in humans," Music Perception, vol. 9, pp. 281-302, Spr 1992.

[17] R. T. Verrillo, "Subjective magnitude functions for vibrotaction," IEEE Transactions on Man-Machine Systems, vol. 11, pp. 19-24, 1970.

[18] P. Richard and P. Coiffet, "Human perceptual issues in virtual environments: sensory substitution and information redundancy.," Robot and Human Communication, 1995. RO-MAN'95 TOKYO, Proceedings, 4th IEEE International Workshop on., pp. 301 - 306, Jul 1995.

[19] C. E. Stepp and Y. Matsuoka, "Relative to direct haptic feedback, remote vibrotactile feedback improves but slows object manipulation," Conf Proc IEEE Eng Med Biol Soc, vol. 2010, pp. 2089-92, 2010.

[20] Qi An; Matsuoka, Y.; Stepp, C.E.; , "Multi-day training with vibrotactile feedback for virtual object manipulation," Rehabilitation Robotics (ICORR), 2011 IEEE International Conference on, vol., no., pp.1-5, June 29 2011-July 12011. 